Spin Physics (SPIN2014)

International Journal of Modern Physics: Conference Series

Vol. 40 (2016) 1660020 (4 pages)

(C) The Author(s)

DOI: $10.1142 / \mathrm{S} 201019451660020 \mathrm{X}$

\title{
Flavour Asymmetry and Strange-Antistrange Asymmetry in the Nucleon Sea
}

\author{
F.-G. Cao \\ Institute of Fundamental Sciences, Massey University, \\ Private Bag 11-222, Palmerston North, New Zealand \\ f.g.cao@massey.ac.nz
}

Published 29 February 2016

\begin{abstract}
We review theoretical calculations for flavour asymmetry and strange-antistrange asymmetry in the nucleon's parton distribution functions using the meson cloud model. We also discuss perturbative mechanism for the generation of strange-antistrange asymmetry in the nucleon.
\end{abstract}

Keywords: Symmetry breaking; Parton distribution function; Meson cloud model.

\section{Introduction}

Since the discovery of flavour asymmetry in the unpolarised proton sea $(\bar{d}>\bar{u})^{1}$ there has been increasing interest in the question of whether this asymmetry extents also to the polarised sea distributions $(\Delta \bar{u}(x) \neq \Delta \bar{d}(x))$ and whether the other symmetries predicted by the parton models (e.g., charge symmetry and quark-antiquark symmetry) are violated as well. Most models proposed in explaining the flavour asymmetry in the nucleon can be extented to make predictions for the breaking of the other symmetries of the parton models. ${ }^{2}$

In the meson cloud model ${ }^{3}$ the nucleon can be viewed as a baryon 'core' surrounded by a mesonic cloud. The lifetime of the virtual baryon-meson components is generally much longer than the interaction time in the deep inelastic process, thus the quarks and antiquarks in the baryon and meson contribute to the parton distribution functions (PDFs) of the nucleon. The observed PDFs are the sum of contributions from the 'bare' nucleon and the virtual baryons and mesons. The contribution from the virtual baryons can be expressed as a convolution of fluctuation function $f_{B M / N}(y)$, which describes the probability for a nucleon fluctuating into a virtual baryon $(B)$ with longitudinal momentum fraction $y$ and a virtual meson $(M)$

This is an Open Access article published by World Scientific Publishing Company. It is distributed under the terms of the Creative Commons Attribution 3.0 (CC-BY) License. Further distribution of this work is permitted, provided the original work is properly cited. 
with longitudinal momentum fraction $1-y$, with the valence PDF of the baryon,

$$
\delta q\left(x, Q^{2}\right)=\int_{x}^{1} \frac{d y}{y} f_{B M / N}(y) q_{B}\left(\frac{x}{y}, Q^{2}\right) .
$$

The contribution from the virtual mesons can be expressed in a similar way.

The flavour asymmetry in the proton $\bar{d}>\bar{u}$ emerges naturally since the probability of finding the $\left|n \pi^{+}\right\rangle$component in the proton is larger than that of the $\left|\Delta^{++} \pi^{-}\right\rangle$ component. The model also predicts violations of SU(3) flavour symmetry, charge symmetry and quark-antiquark symmetry for the parton distribution functions of the nucleon ${ }^{4-} .8$

Apart from the nonperturbative contrbutions to the nucleon sea considered in the moeson cloud model, there are perturbative contributions which arise from gluons splitting into quark-antiquark pairs. The perturbative contributions are generally assumed to obey $\mathrm{SU}(2)$ favour symmetry and quark-antiquark symmetry since the mass difference between up-quark and down-quark and the mass difference between quark and antiquark are negligible. Up to next-to-leading order (NLO) in $\alpha_{s}$ the probability of a splitting $q \rightarrow q^{\prime}$ is identical to that of $q \rightarrow \bar{q}^{\prime}$. A difference between the two probabilities for the two splittings first arises at next-to-next-toleading order (NNLO), which consequently leads to a strange-antistrange asymmetry. ${ }^{9}$ Both nonperturbative and perturbative contributions are included in the calculation for the strange-antistrange asymmetry in Ref. 8.

We review theoretical studies of flavour asymmetry ${ }^{4,5}$ and strange-antistrange asymmetry $^{7,8}$ in the nucleon sea.

\section{Flavour Asymmetry in the Polarised Nucleon Sea}

The calculations for the difference $x[\Delta \bar{u}(x)-\Delta \bar{d}(x)]$ using the meson cloud model are compared with the HERMES measurements ${ }^{10}$ in Fig. 1 . The theoretical calculations are consistent with the data, although large uncertainties exist in the data. We note that the $\mathrm{SU}(2)$ flavour symmetry breaking in the polarised nucleon sea is much smaller than in the unpolarised sea.

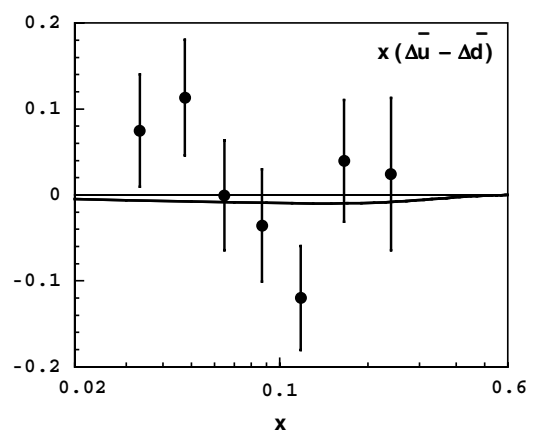

Fig. 1. Flavour asymmetry in the polarised light antiquark sea. 


\section{Strange-Antistrange Asymmetry in the Nucleon Sea}

The perturbative contributions to the asymmetry $x(s-\bar{s})$ are shown in Fig. 2 while the total asymmetry including both perturbative and nonperturbative contributions are shown in Figs. 3 and 4. Only $K$ mesonic cloud was considered in the nonperturbative contributions for Fig. 3, while both $K$ and $K^{*}$ mesonic clouds were considered in the nonperturbative contributions for Fig. 4. Comparing Fig. 2 with Figs. 3 and 4 one can see that the nonperturbative contributions dominate in the region $x>0.1$ while the perturbative contributions dominate in the region $x<0.1$. The nonperturbative contributions are smaller in magnitude than the perturbative contributions, which indicates the asymmetry could be better measured in the region of $x \sim(0.02 \sim 0.03)$.

Figs. 3 and 4 suggest that the asymmetry may have more than one node, in contraction with the common assumption of only one node for the asymmetry used in most global analyses of the PDFs.

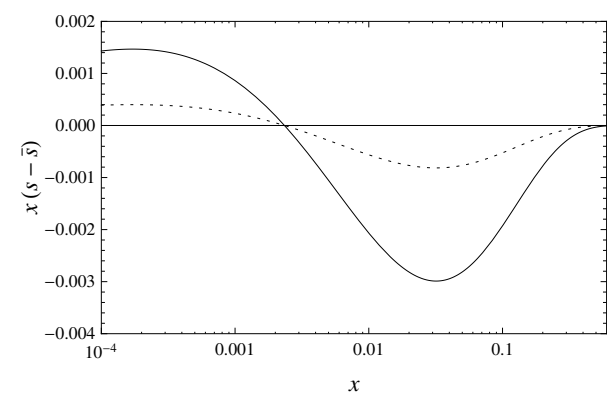

Fig. 2. The perturbative contributions to the asymmetry $x(s-\bar{s})$ at $Q^{2}=16 \mathrm{GeV}^{2}$. The solid and dotted curves are the results calculated with $Q_{0}=0.51 \mathrm{GeV}$ and $Q_{0}=1.1 \mathrm{GeV}$, respectively.

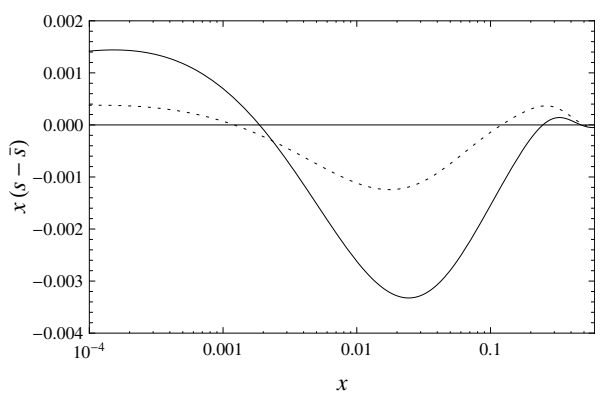

Fig. 3. The total asymmetry $x(s-\bar{s})$ at $Q^{2}=16 \mathrm{GeV}^{2}$ including both the perturbative contributions and nonperturbative contributions which are calculated with only $K$ mesonic cloud. The solid and dotted curves are the results for $Q_{0}=0.51 \mathrm{GeV}$ and $Q_{0}=1.1 \mathrm{GeV}$ being used in the calculations for the perturbative contributions. 


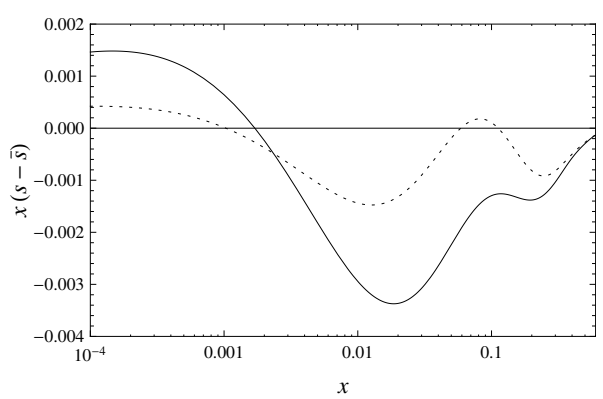

Fig. 4. Similar as Fig. 3. Both $K$ and $K^{*}$ mesonic clouds are considered in calculating nonperturbative contributions to the asymmetry.

\section{Summary}

We review theoretical calculations for the $\mathrm{SU}(2)$ flavour asymmetry and strangeantistrange asymmetry in the nucleon's parton distribution functions. The meson cloud model predicts a smaller $\mathrm{SU}(2)$ flavour asymmetry for the polarised nucleon sea than the unpolarised nucleon sea. There are two mechanisms for the generation of an asymmetry between the strange and antistrange quark distributions in the nucleon: nonperturbative contributions originating from nucleons fluctuating into virtual baryon-meson pairs such as $\Lambda K$ and $\Sigma K$, and perturbative contributions arising from gluons splitting into strange and antistrange quark pairs. While the nonperturbative contributions are dominant in the large- $x$ region, the perturbative contributions are more significant in the small- $x$ region.

\section{References}

1. A. Baldit et al., NA51 Collaboration, Phys. Lett. B 332, 244 (1994); E. A. Hawker et al., E866/NuSea Collaboration, Phys. Rev. Lett. 80, 3715 (1998); K. Ackerstaff et al., HERMES Collaboration, Phys. Rev. Lett. 81, 5519 (1998).

2. For recent reviews, see e.g., W.-C. Chang and J.-C. Peng, Prog. Part. Nucl. Phys. 79, 95 (2014).

3. A. W. Thomas, Phys. Lett. B 126, 97 (1983).

4. F.-G. Cao and A. I. Signal, Eur. Phys. J. C 21, 105 (2001); Phys. Rev. D 68, 074002 (2003).

5. H. Chen, F.-G. Cao and A. I. Signal, J. Phys. G 37, 105006 (2010).

6. F.-G. Cao and A. I. Signal, Phys. Rev. C 62, 015203 (2000).

7. F.-G. Cao and A. I. Signal, Phys. Rev. D 60, 074021 (1999); Phys. Lett. B 559, 229 (2003).

8. G.-Q. Feng, F.-G. Cao, X.-H. Guo and A. I. Signal, Eur. Phys. J. C 72, 2250 (2012).

9. S. Catani, D. de Florian, G. Rodrigo and W. Vogelsang, Phys. Rev. Lett. 93, 152003 (2004).

10. A. Airapetian et al., HERMES Collaboration, Phys. Rev. Lett. 92, 012005 (2004). 